\title{
Intelligent household led lighting system for energy saving.
}

\author{
Miss. Ashwini Deshmukh ${ }^{1}$, Dr. K.B.Khanchandani ${ }^{2}$ \\ ${ }^{I}(E \& t c$, SSGMCE, Shegaon/SGBAU, India) \\ ${ }^{2}(E \& t c$, SSGMCE, Shegaon/SGBAU, India)
}

\begin{abstract}
Daily more and more home appliances and consumer electronics are installed in homes. Residential energy consumption tends to grow rapidly. Around $10 \%$ of a total household power is consumed during standby power mode, the reduction of standby power is greatly necessary to reduce the electricity cost in home. The existing lights cannot be controlled in various ways, it causes unnecessary power consumption. So, the invention of LEDs which have only 50\% of the power consumption as compared to fluorescent is expected to help to lessen the energy consumption problem. So we propose the system in which using different sensors, motion and light is detected and according to those parameters led light is control using wireless communication. So the proposed system saves the energy.
\end{abstract}

Keywords: Lighting control system, Led light, Wireless sensor.

\section{Introduction}

Energy-saving solutions are very essential in recent years because of environmental issues such as climate change and global warming. According to conservation of energy, energy can neither be created (produced) nor destroyed by itself. It can only be transformed. Environmental problems are very important issues and these problems are largely caused by the excessive use of energy. Taking care of the environment is a responsibility that everyone should feel accountable for. Most of us are already aware of environmentally friendly processes such as recycling to minimize the amount of waste we produce and reduce our carbon footprint. However, a lot of people are unaware of new and upcoming technologies that we can use to help reduce carbon emissions. A good example of this is led lightning which provides many environmental advantages. Daily more and more home appliances and consumer electronics are installed in homes. Residential energy consumption tends to grow rapidly. Around $10 \%$ of a total household power is consumed during standby power mode, the reduction of standby power is greatly necessary to reduce the electricity cost in home. LED lights are up to $80 \%$ more efficient than traditional lighting such as fluorescent and incandescent lights. 95\% of the energy in LEDs is converted into light and only 5\% is wasted as heat. This is compared to fluorescent lights which convert 95\% of energy to heat and only 5\% into light! LED lights also draw much less power than traditional lighting; a typical 84 watt fluorescent can be replaced by a 36 watt LED to give the same level of light. Less energy use reduces the demand from power plants and decreases greenhouse gas emissions.

In recent years, usage of LED lights has been increasing. As interest of energy saving broadens, light power consumption, which are one of the highest power consumption, and becomes a big issue. At this point, LED lights will be the most practical answer to decrease power consumption in a home or building. Moreover, LED technology has been researched by many companies and research centers with advanced countries as the center, and now it can be substituted for the existing lights. There are various types from low power to power LED, and it is expected that it will influence lighting markets seriously.Recently, an intelligent lighting control system are developed to reduce energy consumption as automatically controlling the intensity of illumination through situation awareness, such as awareness of user movement or brightness of surroundings. But these lighting control systems can support only on/off or dimming control according to user movement or brightness of surroundings, it is difficult to be applied to complex environments such as warehouse or office. The existing systems are designed without considering user satisfaction. But user's satisfaction is important factor due to saving energy. So the system should be designed considering both energy efficiency and user satisfaction.

\section{Related Work}

Ying-Wen Bai [12] proposed the Home Light Control Module design using a microprocessor and a light sensory for automatic room light detection and control. Presence of human body is sensed by the PIR sensor and accordingly the change of light intensity in a room is sensed by light sensor in the HLCM.

Seung -Ho Hong, [9] carried out an experimental Case Study of a BACnet-Based Lighting Control System providing benefits in building management and stable operation of the control system is guaranteed below a certain threshold of network traffics. The experimental model integrates daylight and fluorescent 
lighting environment. The hierarchical structure of the BACnet building automation system provides extensibility, flexibility and scalability.

Andreas Foglar [1] proposed an architecture designed for Network Energy Technologies for Green WAS provides the home appliances an ability to maintain total energy consumption within User-Defined limits. J.Han, C.S.Choi [4] proposed a Home Energy Management System (HEMS) based on ZigBee Communication and IR remote control system reduces and manage home energy more efficiency by reducing active standby power and controlling the power outlets using ZigBee Hub with IR code learning function.

Johan J. Lukkien [5] proposed an User-Centered Intelligent Road-Lighting Design executing light dimming commands automatically using contextual information extracted from sensory data. Proposed architecture consists of a central terminal and several controllers which uses Power Line Communication for sending commands for sending commands to the Luminary Poles. The PLC infrastructure allows the system to do experiments only at pedestrian speeds not in vehicular speeds.

Aurora Gilde-Castro [10] presents Building Lighting Automation through the Integration of DALI with Wireless Sensor Networks which allows a half-duplex communication which can provide many parameters about the lighting and lamp status. DALI Devices require a dedicated bus for data transmission which results in a reduction of installation cost.

Fabio Leccese [6] presents a Remote-Control System of High Efficiency and Intelligent Street Lighting using a ZigBee Network of Devices and Sensors providing a high efficient and Intelligent Street Lighting. Here highly efficient LED technique supplied by renewable energy of solar panels allows the control system switching on the light only when necessary, increasing the lamp's lifetime so lets the system be suitable for street lighting in urban and rural areas when the traffic in low at a given range of the time.

M.S.Pan, [7] presented A WSN based intelligent light control system considering user activities and profiles in which wireless sensors are responsible for measuring current illuminations and the lights. The lights are controlled by applying the model of user's actions and profiles for indoor environments, such as a home for a reduction in energy consumption.

Jinsung Byun [3] developed a Self-adapting Intelligent System which consists of Self-adapting Intelligent Gateway (SIG) and Self-adapting Intelligent Sensor (SIS) in order to make the consumer devices more energy efficient and intelligent along with the Efficiency Self-clustering Sensor Network (ESSN) and Node Type Indicator Routing Protocol (NTIR) which are introduced to enhance service response time and network lifetime. The middleware inside microprocessor memory is dynamically reconfigured and modified to enhance QoS such as the service response time, hit ratio, situation analysis and the energy saving performance.

Young-Sung Son [17] describes a home energy management system based on power line communication that can provide easy-to-access information on home energy consumption in real time, intelligent planning for controlling appliances, and optimization of power consumption at home. Smart metering and power line communication can provide detailed information of energy consumption patterns and intelligent controlling to appliances at home. Especially, planning makes to shift device controlling on peak price time and to smooth power demand work load providing benefits about resource utilization, energy conservation and cost reduction to users.

Cheng-Hung Tsai, Ying-Wen Bai, [18] presented a way to reduce the standby power of a socket. The socket supplies appliances with power when the user turns them on. System design uses an MCU, receives signals from a PIR sensor which detects the user approaching the socket. The MCU controls the SSR On/Off when used as an appliance switch for shutting off the standby power. The PV array is added in design to reduce the consumption from the local electric power company.

Byoungjoo Lee, Insung Hong, [8] designed and Implemented a power aware LED light enabler with light sensors, motion sensors and network interfaces which communicates with context- aware middleware using an intelligent power gateway that adoptively determines the optimal power control by analyzing user living patterns. The proposed middleware facilitates the learning mechanism which analyzes the illumination and the user activity and controls the LED lights only when users exists around the devices.

The lighting control system for energy savings in current markets can support on-off and dimming control as managing lighting devices after detecting an object or intensity of Illumination, or controlling with time setting. Furthermore, although most existing systems have variable control parameters, it is difficult for users to modify these parameters, so that it is not appropriate to be applied in various places. These systems are not convenient for users because of frequent light on/off.

\section{Working Principle}

Intelligent Energy Saving System, the aim of the project is to save the energy. In this project we are using various sensors, controlling and display.

In this project work the basic signal processing of various parameters which are, LDR, motion sensor. For measuring various parameters values, various sensors are used and the output of these sensors are converted 
to control the parameters. The control circuit is designed using micro-controller. The outputs of all the three parameters are fed to micro-controller. The output of the micro-controller is used to drive the led light as shown in "Fig." 1.1. Bluetooth is used to control intensity of led light. The proposed LED system can autonomously adjust the light intensity value to enhance both energy efficiency and user satisfaction.

The proposed system provides intelligent mechanism for effective energy management using multi sensors and wireless communication technology to control LED light according to user's state and surroundings. We use environmental sensors like illumination sensors (LDR), motion sensor (PIR), which allows the system to vary brightness of illumination. The system using PIR sensor decides whether a user is in a room or not and control the brightness of lights connected with microcontroller. The microcontroller continuously monitors the sensors. In LED lights, it is possible to adjust the brightness of the lights by using PWM. i.e. if the illumination of surrounding is sensed, uses can be provided the regular brightness through brightening or darkening the lights freely. Also it varies the illumination according to the insulation of the season or the time of day. When the movements are rarely detected, system autonomously turns the LED lights off or adjusts the weaker brightness to save power consumption.

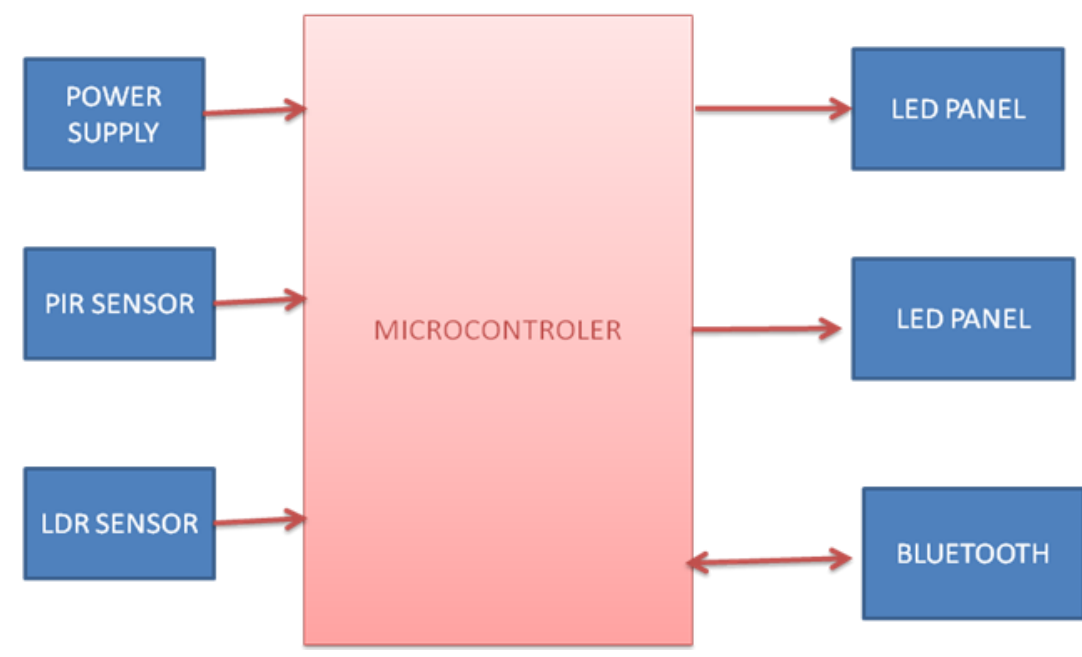

Fig 1 .Block diagram

\section{Pir sensor}

\section{Components}

A PIR detector is a motion detector that senses the heat emitted by a living body. These are often fitted to security lights so that they will switch on automatically if approached. They are very effective in enhancing home security systems. The sensor is passive because, instead of emitting a beam of light or microwave energy that must be interrupted by a passing person in order to "sense" that person, the PIR is simply sensitive to the infrared energy emitted by every living thing. When an intruder walks into the detector's field of vision, the detector "sees" a sharp increase in infrared energy.

\section{Ldr sensor}

LDR's or Light Dependent Resistors are very useful especially in light/dark sensor circuits. These help in automatically switching ON /OFF the street lights and etc., normally the resistance of an LDR is very high, sometimes as very high as 1000000 ohms, but when they are illuminated with light, resistance drop dramatically. Electronic opto sensors are the devices that alter their electrical characteristics, in the presence of visible or invisible light. The best-known devices of these types are the light dependent resistor (LDR), the photo diode and the phototransistors.

\section{Bluetooth serial interface module}

Bluetooth serial module is used for converting serial port to Bluetooth. These modules have two modes: master and slaver device. The device named after even number is defined to be master or slaver when out of factory and can't be changed to the other mode. But for the device named after odd number, users can set the work mode (master or slaver) of the device by AT commands. During the pairing, the current is fluctuant in the range of $30-40 \mathrm{~m}$. The mean current is about $25 \mathrm{~mA}$. After paring, no matter processing communication or not, the current is $8 \mathrm{~mA}$. There is no sleep mode. This parameter is same for all the Bluetooth modules. 
Intelligent household led lighting system for energy saving.

\section{Arduino}

The Arduino Uno is a microcontroller board based on the ATmega328. It has 14 digital input/output pins (of which 6 can be used as PWM outputs), 6 analog inputs, a $16 \mathrm{MHz}$ crystal oscillator, a USB connection, a power jack, an ICSP header, and a reset button. It contains everything needed to support the microcontroller; simply connect it to a computer with a USB cable or power it with a AC-to-DC adapter or battery to get started. The Uno differs from all preceding boards in that it does not use the FTDI USB-to-serial driver chip. Instead, it features the Atmega8U2 programmed as a USB-to-serial converter.

\section{Results}

Table 1 .Energy Consumption of Appliances

\begin{tabular}{|l|l|l|l|l|l|}
\hline \multirow{3}{*}{ Days } & \multicolumn{2}{|l|}{ On time of the Appliances (hrs) } & \multicolumn{2}{|l|}{ Energy Consumption (W/hrs) } & \multirow{2}{*}{ Total Energy Saved (W/hrs) } \\
\cline { 2 - 5 } & $\begin{array}{l}\text { With Developed } \\
\text { Hardware }\end{array}$ & $\begin{array}{l}\text { Without Developed } \\
\text { Hardware }\end{array}$ & $\begin{array}{l}\text { With Developed } \\
\text { Hardware }\end{array}$ & $\begin{array}{l}\text { Without Developed } \\
\text { Hardware }\end{array}$ & 369.6 \\
\hline Sun & 7.92 & 11.00 & 950.4 & 1320.0 & 414.0 \\
\hline Mon & 6.55 & 10.00 & 786.0 & 1200.0 & 372.0 \\
\hline Tues & 7.15 & 10.25 & 858.0 & 1230.0 & 218.4 \\
\hline Wed & 7.40 & 9.22 & 888.0 & 1106.4 & 404.6 \\
\hline Thurs & 6.33 & 9.70 & 759.0 & 11.64 .0 & 506.4 \\
\hline Fri & 12.40 & 16.62 & 1488.0 & 1994.0 & 267.6 \\
\hline Sat & 10.15 & 12.38 & 1218.0 & & \\
\hline
\end{tabular}

\section{Conclusion}

The proposed system utilizes multi sensors and wireless communication technology in order to control an LED light according to the user's state and the surroundings. The proposed system can autonomously adjust the minimum light intensity value to enhance both energy efficiency and user satisfaction. With the increasing cost of conventional energy, this system is an efficient means of saving energy. The power supply used, is from renewable source which helps in reducing dependence on conventional electricity. Intelligent Energy Saving System is not limited for any particular application, it can be used anywhere in a process industries with little modifications in software coding according to the requirements.

\section{PAPERS}

\section{References}

[1]. Andreas Foglar, Halid Hrasnica, Maurice Draaijer, Nikolaos Mouratidis, Spyridon Tompros , "Enabling Applicability of Energy Saving Applications on the Appliances of the Home Environment", November/December 2009, pg no.8-16.

[2]. Haesik Kim, Honggang Zhang, Kari Horneman, Tao Chen, Yang Yang," Network Energy Saving technologies for Green Wireless Access Networks", October 2011, pg no. 30-38.

[3]. Jinsung Byun, Sehyun Park," Development of a Self-adapting Intelligent System for Building Energy Saving and Context-aware Smart Services", Vol. 57, no.1,February 2011, pg no.90-98.

[4]. Jinsoo Han, Chang-Sic Choi, and Ilwoo Lee, "More Efficient Home Energy Management System Based on ZigBee Communication and Infrared Remote Controls",Vol.57, no.1 February 2011,pg. no. 85-89.

[5]. Cagdas Atici, Tanir ozcelebi and Johan J. Lukkien, "Exploring User-Centered Intelligent Road Lighting Design A Road Map and Future Research Directions", Vol.57, no.2, February 2011,pg. no. 788-793.

[6]. Fabio Leccese, "Remote-Control System of High Efficiency and Intelligent Street Lighting Using a ZigBee Network of Devices and Sensors", Vol.28, no.1, January 2013,pg. no.21-28.

[7]. Meng-Shiuan Pan, Lun-Wu Yeh, Yen-Ann Chen, Yu-Hsuan Lin, and Yu-Chee Tseng, "A WSN-based Intelligent Light Control System Considering User Activities and Profiles", pg. no.1-12.

[8]. Byoungjoo Lee, Gwanyeon Kim, Insung Hong, Sehyun Park, Yoonsik Uhm, “ Design and Implementation of Power-aware LED Light Enabler with Location-aware Adaptive Middleware and Context-aware User Pattern”, January 2010,pg no.231-239.

[9]. Seung-Ho Hong, Tae-Jin Park,'Experimental Case Study of a BACnet-Based Lighting Control System”, Vol. 6, no. 2, April 2009, pg no. 322-333.

[10]. Aurora Gil-de-Castro and Antonio Moreno-Munoz, Francisco Domingo-Perez, Francisco Jose Bellido- Outeirino, Jose Maria Flores-Arias,"Building Lighting Automation through the Integration of DALI with Wireless Sensor Networks", 2012, pg no.47-52.

[11]. Author Name: A. Schoofs, A. Guerrieri, A.G. Ruzzelli, G.M.P. O’Hare," ANNOT: Automated Electricity Data Annotation Using Wireless Sensor Networks “, June, 2010.

[12]. Ying-Wen Bai and Yi-Te Ku, "Automatic Room Light Intensity Detection and Control Using a Microprocessor and Light Sensors", Vol. 54, No. 3, August 2008, pg. no. 1173-1176.

[13]. R.A.Ramlee, M.H.Leong, R.S.S.Singh, M.M.Ismail, M.A.Othman, H.A.Sulaiman, M.H.Misran, M.A.Meor Said, "Bluetooth Remote Home Automation System Using Android Application", The International Journal of Engineering And Science,Vol. 2,no.1, 2013, pg. no. 149-153.

[14]. Hayoung Oh, Hyokyung Bahn, and Ki-Joon Chae, "An Energy-Efficient Sensor Routing Scheme for Home Automation Networks", Vol. 51, No. 3, August 2005, pg. no.836-839.

[15]. Jinsoo Han, Haeryong Lee, and Kwang-Roh Park, "Remote-Controllable and Energy- Saving Room Architecture based on ZigBee Communication", Vol. 55, No. 1, February 2009, pg. no.264-268. 